\title{
Lateral foot pain
}

\author{
Diagnosis : Iselin's disease
}

\author{
Hatice Tuba Sanal ${ }^{1} \cdot$ Lina Chen ${ }^{2}$
}

Published online: 31 May 2015

(C) ISS 2015

Dr. Iselin was the first to describe the entity of traction apophysitis of the secondary ossification center of the fifth metatarsal base in 1912 [1]. The apophysis can be considered a "bridge" between tendon and bone, as in this case, the peroneus brevis tendon and the fifth metatarsal base [2]. The apophysis is susceptible to trauma and chronic stress [2]. Apophysitis is thought to result from repetitive trauma, with initial interruption of the blood supply resulting in avascular necrosis, followed by gradual resorption of dead bone with regeneration and recalcification, seen radiologically as fragmentation $[2,3]$. The diagnosis of apophysitis was given initially to patients who presented in clinics with pain and swelling [1-3]. However, subsequently, the underlying pathogenesis was found to be non-inflammatory, therefore the term apophysitis may be misleading. Some authors suggest calling these chronic repetitive stress injuries rather than apophysitis $[4,5]$. The previously reported cases are radiographs with a spectrum of appearance from a small fleck of bone to fragmentation [2]. Magnetic resonance (MR) imaging early on in the disease process may show bone marrow edema, as in this case before frank fragmentation occurs. It is the bone marrow edema within the apophysis and sometimes reactive soft tissue edema that are suggestive of the diagnosis. In addition to increased signal intensity of the apophysis on fluid sensitive sequences, physeal widening and associated muscle edema

The case presentation can be found at doi: 10.1007/s00256-015-2180-y

Hatice Tuba Sanal

tubasanal@gmail.com

1 Radiology Department, Gulhane Military Medical Academy, Ankara, Turkey

2 Radiology Department, University of Maryland School of Medicine, Baltimore, MD, USA

may be observed. MR imaging can be useful to make the diagnosis when radiographic findings are unrevealing and in athletes prior to recommending return to play.

The mimickers of avulsion and Jones fractures can be differentiated from Iselin's disease (ID) via their location and orientation on radiographs: $i$ ) avulsion fracture being transverse in orientation; ii) Jones fractures being at the metadiaphyseal junction more distally; and iii) ID occurring at the normal growth plate appearing as a longitudinal lucent line parallel to the long axis of the fifth metatarsal shaft [6]. Another differential consideration is os vesalianum pedis, an uncommon accessory ossicle located proximal to the base of the fifth metatarsal, usually seen as a well-corticated rounded ossicle articulating with the proximal fifth metatarsal $[6,7]$.

Conflict of interest The author's declare that they have no competing interests.

\section{References}

1. Ruci V, Duni A, Serani D. Iselin' disease - apophysitis of the fifth metatarsal base: report of two cases. Maced J Med Sci. 2013;6:4379 .

2. Ralph BG, Barrett J, Kenyhercz C, DiDomenico LA. Iselin's disease: a case presentation of nonunion and review of the differential diagnosis. J Foot Ankle Surg. 1999;38:409-16.

3. Hunter LY, O'Connor GA. Traction apophysitis of the olecranon. Am J Sports Med. 1980;8:51-2.

4. Hébert KJ, Laor T, Divine JG, Emery KH, Wall EJ. MRI appearance of chronic stress injury of the iliac crest apophysis in adolescent athletes. AJR Am J Roentgenol. 2008;190:1487-91.

5. Pointinger H, Munk P, Poeschl GP. Avulsion fracture of the anterior superior iliac spine following apophysitis. Br J Sports Med. 2003;37: $361-2$.

6. Deniz G, Kose O, Guneri B, Duygun F. Traction apophysitis of the fifth metatarsal base in a child: Iselin's disease. BMJ Case Rep. 2014. doi:10.1136/bcr-2014-204687.

7. Kose O. Os vesalianum pedis misdiagnosed as fifth metatarsal avulsion fracture. Emerg Med Australas. 2009;21:426. 